\title{
S41. Novel CEA-targeted IL2 variant immunocytokine for immunotherapy of cancer
}

\author{
C Klein \\ From 1st Immunotherapy of Cancer Conference (ITOC1) \\ Munich, Germany. 12-14 March 2014
}

\section{Background}

Here we describe a novel class of monomeric tumortargeted immunocytokines that comprise a single IL-2 variant (IL2v) with abolished CD25 binding that is fused to the C-terminus of a tumor specific antibody with a heterodimeric Fc devoid of Fc $\gamma \mathrm{R}$ and $\mathrm{C} 1 \mathrm{q}$ binding. For tumor targeting, human/humanized high affinity antibodies against CEA (GA504) or FAP (GA501) were selected.

\section{Materials \& methods}

CEA- and FAP-IL2v activity was tested on effector cells by assessing the activation of P-STAT5, cell proliferation, sensitivity to Fas-induced apoptosis, expression of activation markers and cytokine release upon treatment. Safety, pharmacokinetics, pharmacodynamics and anti-tumor efficacy were analyzed in fully immunocompetent (CEA transgenic) $\mathrm{C} 57 \mathrm{Bl} / 6$ mice as single agent and in combination with ADCC competent antibodies in SCID/hCD16 tg mice as well as Balb/neuT genetically engineered mice. Tumor targeting was investigated in the orthotopic syngeneic Renca RCC model in Balb/c mice.

\section{Results}

FAP- and CEA-IL2v completely lack binding to CD25, but retain IL-R $\beta \gamma$ binding, and show $\mathrm{pM}$ binding affinity to respective antigens, FAP or CEA. As a consequence of abolished binding to CD25 they do not preferentially activate Tregs, but IL-2R $\beta \gamma$ mediated activity is retained and FAP- and CEA-IL2v activate NK, CD $4^{+}$and $\mathrm{CD} 8^{+} \mathrm{T}$ cells as shown by induction of activation markers, cell proliferation and cytokine release. Furthermore, CEA-IL2v and FAP-IL2v enhance the cytotoxic activity of NK cells when combined with ADCC-competent antibodies. Mechanism of action studies in fully immunocompetent mice showed that the molecules strongly expand and activate $\mathrm{NK}, \mathrm{CD} 8^{+}$
T cells and gd T cells (up to 100-fold) and skew the CD4: $\mathrm{CD} 8$ ratio strongly towards $\mathrm{CD}^{+} \mathrm{T}$ cells in the peripheral blood, lymphoid tissues, and in the tumor. In C57Bl/6 mice. MicroSPECT/CT imaging with radioactively labeled FAP-IL2v reveal good FAP-mediated tumor targeting in the orthotopic syngeneic Renca model with low normal tissue uptake and low accumulation in lymphoid tissues, contrary to analogous IL-2 based immunocytokine that shows preferential targeting to lymphoid tissue. Studies in tumor-bearing mice show dose-dependent anti-tumor efficacy of CEA-IL2 $v$ in syngeneic MC38-CEA and PancO2CEA models. Combination of a tumor-stroma targeted TNCA-IL2v with an ADCC-competent ratHER2 antibody in the Balb/neuT spontaneous breast cancer model results in enhanced antitumoral efficacy.

\section{Conclusion}

Compared to classical IL-2-based immunocytokines, CEAand FAP-IL2v demonstrate superior safety, PK and tumor targeting due to abolished CD25 binding, monovalency and high-affinity to tumor antigens while failing to preferentially induce Tregs. CEA- and FAP-IL2v retain the capacity to activate and expand NK and CD8+ effector T cells both in the periphery and tumor microenvironment supporting their further nonclinical and clinical investigation for immunotherapy of cancer. Clinical trials with CEAIL2v are foreseen in 2014.

Published: 12 March 2014

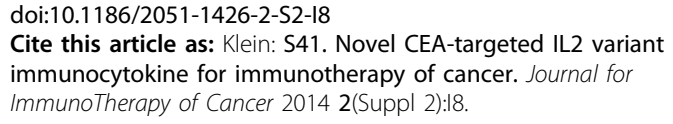

